\title{
Time determination of permanence of the sengstaken blakemore ballon in relation with the clinical outcome in postpartum hemorrhage patients
}

\begin{abstract}
Postpartum hemorrhage is one of the leading causes of maternal death in the world, representing the $27 \%$. More than two thirds of these cases have been classified as postpartum hemorrhage (PPH). The guidelines for the treatment of PPH implicate a step by step focus, initiating with the exclusion of retained placental tissue, genital tract trauma, uterine massage and multiple uterotonic agents. If bleeding control is not accomplished, it requires surgical intervention like compression sutures, internal iliac artery ligation and obstetric hysterectomy. Recently the balloon uterine tamponade has been added to the management of PPH. Here in the High Specialty Unit we use the Sengstaken Blakemore (SSB) balloon as uterine tamponade.

Objectives: Comparing the time of the SSB balloon permanence in relation to the bleeding control success in postpartum hemorrhage patients.

Methods: A comparative, retrospective and transversal analytical study will be carried out in patients that had there pregnancy terminated in this unit and was treated with SSB uterine tamponade.

Results: Registries were obtained from 149 files from patients with PPH that were treated with SSB uterine tamponade in a period of time understood from 2013-2014, a success rate was found with SSB uterine tamponade of $92.62 \%(\mathrm{P}=<0.001)$, it was estimated that over 20.5 hours (SEN 52.9\%, SPE 77.2\%) doesn't offer greater benefit carrying out the permanence of the SSB uterine tamponade, securely removing the tamponade at this time.

Conclusion: The SSB uterine tamponade is being used in Monterrey, Nuevo Leon with a greater success rate than the one reported in literature; the age of the patient and the number of pregnancies are variables that can predict the probable success of the treatment, and that once installed the ideal time of permanence of the uterine tamponade can be less than 20.5 hours.
\end{abstract}

Volume 9 Issue I - 2018

\author{
Yolanda Reynosa Oviedo,' Ernesto Arnoldo \\ Lopez Vera, ${ }^{2}$ Salomon Alvarado, ${ }^{3}$ Alma Karol \\ Urquidez Acosta,' Nefi Salazar Hernandez ${ }^{4}$ \\ 'Surgeon of the Immediate Response Team, High Specialty \\ Medical Unit 23 Hospital of Gynecology and Obstetrics, Mexico \\ ${ }^{2}$ Surgeon in Chief of the Immediate Response Team, High \\ Specialty Medical Unit 23 Hospital of Gynecology and \\ Obstetrics, Mexico \\ ${ }^{3}$ Anesthesiologist of the Immediate Response Team, High \\ Specialty Medical Unit 23 Hospital of Gynecology and \\ Obstetrics, Mexico \\ 4Second Year OB/GYN Resident, High Specialty Medical Unit 23 \\ Hospital of Gynecology and Obstetrics, Mexico
}

\begin{abstract}
Correspondence: Yolanda Reynosa Oviedo, Surgeon of the Immediate Response Team, High Specialty Medical Unit 23 Hospital of Gynecology and Obstetrics "Dr. Ignacio Morones Prieto" Monterrey, Nuevo Le $\square$ n, Mexico, Emailyolita66@hotmail.com
\end{abstract}

Received: December 22, 2017 | Published: February 6, 2018

\section{Background}

In 2010 it was estimated an incidence of 287,000 cases of maternal death worldwide, the majority of this cases were evitable and were registered in low income countries. The reduction of maternal death has been a global priority for a long time and its one of the Millennium Development Goals and one of the major concerns of the World Health Strategy for Women and Children launched by the Secretary General of the United Nations Organization on September 2010. In 2006, it was determined that the hypertensive disorders and postpartum hemorrhage were the leading causes of maternal death in developing countries. Recently, the Global Burden Disease Study (GBD) gave estimations of the major causes of maternal death, while the deaths of indirect causes represented $27.5 \%$. Postpartum Hemorrhage is the most frequent and direct cause in the world representing $27 \%$. More than two thirds of these cases were classified as postpartum hemorrhage. Hypertension is the second direct cause of the in the world, with $14.0 \%$. Maternal Death due to sepsis was $10.7 \%$, abortion represented the $7 \%$ and other causes represented the rest with $12.8 \%$.

Despite the interventions stablished to prevent and treat postpartum hemorrhage (like the active management of the third period of labor), postpartum hemorrhage is still the leading individual cause of maternal death. It hasn't been possible to stablish if the persistence of the hemorrhage is the leading cause despite effective interventions, it is the result of a lack of applications of well stablished measures. If there is a change towards the hemorrhage before delivery or a different way of terminating the pregnancy. ${ }^{1}$

Postpartum Hemorrhage (PPH) is defined as a bleeding of more than $>500 \mathrm{ml}$ after vaginal delivery and $1000 \mathrm{ml}$ after cesarean delivery in the first $24 \mathrm{hrs}$. The most common cause is uterine atony responsible for $80 \%$ of the cases. Other causes of postpartum hemorrhage are retained placental tissue, uterine rupture, obstetric trauma, uterine inversion and coagulopathy. The guidelines for postpartum hemorrhage implicate a step by step focus, initiating with the exclusion of retained placental tissue and obstetrical trauma. Uterine Atony is treated with uterine massage and compression and multiple uterotonic agents such as oxytocin, ergonovine, misoprostol and F2 a Prostaglandins (PGF2a). If bleeding control is not accomplished, it requires surgical intervention like compression sutures, internal iliac artery ligation and obstetric hysterectomy. Recently the balloon uterine tamponade has been added to the management of $\mathrm{PPH}$. Although Uterine Tamponade has recently been added to the management of PPH by the WHO in 2012 as a rescue intervention for PPH, it hasn't been integrated to the Health Systems Protocols. ${ }^{2}$ In the majority of the Health Centers this procedure has been used before surgical interventions. Historically uterine tamponade was used with gauzes and compresses, but the technical difficulty of insertion and the risk of perforation, the SSB uterine tamponade have been favored 
by gynecologists. In various series of studies, it has been reported the successful use of a great variety of balloon devices, including the Sengstaken - Blakemore balloon, Bakri SOS Balloon, Rush, Foley catheters and condoms. As this is a less invasive method and faster method it would be logical to use it as the first step of treatment after the failure of medical treatment and before proceeding to the surgical treatment and possible hysterectomy. Other advantages that this method offers are avoiding laparotomy, easy and fast insertion and the fact that can be placed by low experienced personal the extraction is painless and offers a rapid way to evaluate the effectiveness of the balloon on failure attempts. As it is a simple procedure to make it can be made by First Level of Attention personal before referring a patient to another unit, once the SBB Uterine tamponade is placed, it will minimize blood loss and will avoid irreversible shock and mortality. ${ }^{3,4}$ The condom associated to a catheter (Bangladesh) adjusts naturally to the uterine form and exercises a uniform pressure over the venous sinuses in the uterine vasculature which stops the hemorrhage. It insufflates until the bleeding stops and generally $250-300 \mathrm{ml}$ of saline solution are enough, which flows freely at the beginning and slows at the end. This contrasts with the reduction of bleeding. It doesn't require a specific anesthetic, but analgesia can be used. Rajni Lohano et al. ${ }^{1}$ informed success rate of $90.4 \%$ for the control of postpartum hemorrhage with condom associated to a catheter (BANGLADESH). Alaska Pendleton et al. ${ }^{4}$ designed and implemented a kit in Kenya, consisting of a syringe, Luer closing valve and a condom strapped at the bottom of a Foley catheter with a cotton thread, the device can be inflated with any solution throughout a syringe of $60 \mathrm{ml}$ and is inserted to the uterine cavity. The kit includes a 3 hours training of the management of PPH. To September 2015 these kits were applied in eight developing countries on the past three years. During the Kit Training it was instructed that the uterine balloon was to be used as a rescue device when PPH was refractory to medical treatment and other measures. Although previous studies have shown that UBT can be applied with efficiency in cases of PPH to avoid maternal death, there are no previous studies that have examined the impact of the decisions of placing a UBT rather than surgical management. ${ }^{2}$

The Bakri Balloon is a intrauterine device indicated to reduce or control PPH temporally when the conservative management is justified. It seems to be a rather efficient alternative for PPH acute management it requires a minimal training to be used. The Bakri Balloon constitutes of a silicone based balloon connected to a catheter of the same material, with a total length of $58 \mathrm{~cm}$ and a total capacity of $800 \mathrm{ml} .{ }^{3,5}$ It has been recommended that we use $250-500 \mathrm{ml}$, depending on the size and uterine capacity. ${ }^{6,7}$ The collapsed balloon is inserted in the uterine cavity and when inflated with liquid it adjusts to the form of the uterus and stops the hemorrhage, it has been reported that the location in the uterine segment and the uterine isthmus, compressing the uterine arteries this position may be considered to be mistaken by some. Nevertheless authors point that untreatable bleeding was controlled successfully and that this could be another mechanism of action of the balloon. ${ }^{6,7}$ Drains of blood throughout the catheters central lumen allows the blood loss to be evaluated. While the balloon stays in the uterine cavity, oxytocin must be continued on continuous infusion for 24 hours. Carbetocine is an oxytocin analogous that has a longer half-life than oxytocin (4-10 times compared with oxytocin), so it can be the drug of choice to achieve contraction for long periods of time when the uterine balloon is placed. ${ }^{6}$ While using the balloon is important to reduce the risk of iatrogenic infection by vaginal bacteria that can produce contamination. Various publications suggest that a first-generation cephalosporin can be the antibiotic of choice. The duration of this can be prophylactic or continuous for 24 until
48 hours while the balloon is in the uterine cavity. ${ }^{6}$ The major virtues that describe the Bakri Balloon are ease of insertion (transvaginal or transabdominal), rapid uterine tamponade, simplify hemorrhage control and to avoid the necessity to use other invasive methods. One of the biggest series reported that the balloon was successful in $90 \%$ of the cases and for PPH caused by atony in a $100 \%$. The original study made by Bakri, 3-5 cases with placenta Previa and low insertion placenta were successfully treated with just the Bakri Balloon while the other two were not. These two patients were treated with Internal Iliac Artery ligation. In the study made by Nagai et al. ${ }^{2}$ the success rate was of $90 \%$ and for PPF caused by atony was of $80 \%$, in these the medium of volume of solution was $200 \mathrm{ml}(300-150 \mathrm{ml})$.

The median duration of permanence of UTB with Balloon was of 24 hours, although it has been described that the duration of the UTB SOS Bakri depending on the case and the quantity of blood loss that passes throughout the drain lumen, it seems to be necessary at least of 12 hours. ${ }^{5}$ In the majority of the reports the balloon has been removed in 24 hours with a $20 \mathrm{ml} / \mathrm{hr}$ rate of deflate until the half of the volume in 12 hours. ${ }^{6}$ It is not clear if the UTB Bakri SOS is efficient for retained placenta or placental abruption. As for placental retention, a previous study demonstrated that the Bakri Balloon was efficient in 12 of 15 patients $(80 \%)$. Also, it was found in literature a successful case of UTB with Bakri Balloon in a patient with retained placenta along with atony. ${ }^{8}$ As for Disseminated Intravascular Coagulopathy (DIC), due to abrupt placentae, the efficiency of the Bakri Balloon has not yet been established. Anti DIC therapy and blood transfusion are the best treatment for bleeding due to abrupto placentae. Nevertheless, UTB with Bakri Balloon can give enough time to prepare other hemostatic interventions. The Bakri SOS Balloon seems to be contraindicated in cases of uterine rupture, because one of its mechanisms is to raise the uterine pressure and rigidity. It has been attributed few adverse effects with the Bakri Balloon. It has been shown; endometritis, fever, balloon displacement, pain, uterine rupture, balloon damage. ${ }^{9-11}$ In the cohort study made in Nuevo Leon in 2013, it is reported an efficiency percentage of more than $90 \%$ with the Sengstaken Blakemore Balloon, the major indication for application was uterine atony, mainly used in multigravida women, and the mean time of permanence of the UTB was 20 hours (less than the literature reports) and also less packed red blood cell were used than in other studies, attributed to the ability of the personel to identify the necessity of UTB, application technique and efficiency. ${ }^{12}$

In the series described by Salih Burçin Kavak, was used a catheter with double balloon in 7 patients. The catheter was applied with double balloon to all patients, and was inflated in the uterine part with saline solution with a quantity that oscillated between $80-110 \mathrm{ml}$ and the vaginal part with $80 \mathrm{ml}$ because the contraction of the uterus was enough. The hemorrhage was controlled with success and they didn't require any other intervention. They concluded that the UTB with double balloon can control efficiently the bleeding that originates in the inferior parts of the uterus and superior parts of the vagina. They recommend the use of the balloon to stop the bleeding in the inferior parts of the uterus, but the Bakri Balloon is more appropriate for uterine atony thanks to its diameter. Also, the double balloon catheter can be preferred when the cervix is so small that the Bakri Balloon can't be inserted throughout the cervix. ${ }^{13}$

When all first line treatments fail, second line therapies are used, including surgery, interventional radiology or treatments such as Recombinant Factor VII (rFVIIa). Hysterectomy is the definite measure to control bleeding. This procedure is related with serious complications such as major blood loss, greater hospital stay, risk of 
organ damage (urinary tract, gastrointestinal) hence the importance of looking for other noninvasive therapies to treat this patients. ${ }^{14}$

In a national cohort of United Kingdom published by Kayem et al., ${ }^{14}$ in 2001 it is described a second line therapy ratio of 2,2 cases for every 10,000 (approximately 1 of every 4500 ) women that gave birth. (CI of 95\%: 1.9-2.5/10000). $73 \%$ of patients were treated with uterine compression sutures, $7 \%$ with pelvic arteries ligation, $8 \%$ with interventional radiology technique and $11 \%$ received rfVIIa. The hysterectomy ratio was of $26 \%$ (CI of $95 \%$ : 19-34). Uterine Compression Sutures had a success of $75 \%$; CI of $95 \%$ : $67-81 \%$. Pelvic Artery ligation had a 36\% success, CI of 95\%: $13-65 \%$. The interventional radiology procedures had a rate of success of $86 \%$; CI of $95 \% 57-98 \%$. The rFVIIa had a $31 \%$ success, CI of $95 \%$ : $11-$ $59 \%$. The success rates were not significantly different when these therapies were used after the failure of UTB. The study shows that even after these interventions, nearly one fourth of the women that had a hysterectomy for final control of the hemorrhage. ${ }^{15}$

Internal Iliac Artery Ligation has been defined as an efficient method to control untreatable PPH and prevention of maternal death. The justification for these stands on the Burchel hemodynamic studies that showed a $45 \%$ reduction on the pelvic blood flow and the pulse pressure on an $85 \%$, which results in increase on the blood venous pressure in the arterial circuit promoting hemostasis. Nevertheless, the rate of success of IIAL varies from $40-100 \%$ and the procedure avoids hysterectomy on $50 \%$ of the cases. It is believed that is a difficult procedure but is much faster than a hysterectomy and rarely tried. ${ }^{15}$

\section{Problem statement}

\section{Magnitude}

The UTB has been established more and more since its introduction by the WHO in 2012, with the only purpose to avoid any surgical intervention. It's a less invasive and faster insertion method that with a minimum of analgesia can be done by less experience personal, commonly the extraction is painless and the identification of failed cases is rapid. Because it's a procedure easy to carry out, the first contact personnel can do it before referring the patients to a more specialized unit that would minimize the blood loss and would prevent inevitable shock and mortality, for this reasons is logic to use it as the first step of management after the failure of the medical treatment, also has demonstrated to be of use for improving the hemodynamic conditions of the patients, meanwhile it prepares for the realization of surgery on the next following hours, for example hysterectomy. Many types of balloons have been described for UTB, among them the Sengstaken Blakemore Catheter, Bakri SOS, Rush Balloon, Foley Catheters and Condoms (Bangladesh). The most studied is the Bakri Balloon, with a success rate greater than $90 \%$ in the majority of the series. In our hospital, we use the Sengstaken Blakemore Balloon as $\mathrm{UTB}$, is the first step of the second line treatment of PPH after the medical treatment failure, which has an efficiency rate greater than $90 \%$.

\section{Trascendence}

There are multiple studies that show different points to take in account in the application and permanence of such balloons in relation of the obtained success, as the amount of solution from which is inflated, the quantity of blood from the drain since its application, cause of hemorrhage, required blood transfusions if the patient is multigravida or not, even the rate of deflated solution, however there are no studies that demonstrate the ideal time of permanence of the balloon that assures the success of the balloon.

\section{Vulnerabilty}

In this study we will determine the time of permanence of the balloon, according with the clinical outcome of the patients, from which would be possible to answer the investigation question.

Is it possible to determine the adequate time of permanence of uterine tamponade with Sengstaken Blakemore Balloon in Postpartum Hemorrhage patients?

\section{Justification}

Based on the actual evidence in the literature, there is no precise time of permanence of the SSB UTB, having our unit as a parameter, multiple managements are registered on the medical personal that put to manifest that even internally, it does not exist a consensus, so doing an exploration of the clinical outcome of the patients that were submitted to such procedure, the success or the application and the time of permanence of the SSB UTB is important for future recommendations on this matter.

\section{Nule hypothesis}

There are no differences in the time of permanence of the SSB UTB between patients that have success in the control of the bleeding and those that were therapy fails.

\section{Altern hypothesis}

There are differences in the time of permanence of the SSB UTB between patients that have success in the control of the bleeding and those that were the therapy fails.

\section{Objectives}

General objective: Compare the times of permanence of the SSB Balloon in patients with postpartum hemorrhage in relation to the success of the control of the bleeding

\section{Specific objectives:}

a. Describe the sociodemographic, anthropometric and gynecological background in the patients included in the study.

b. Observe and describe characteristics of the population that modify the outcome of the treatment

c. Detect risk of factors that have clinical impact on the application of the uterine tamponade.

d. Determine the time of permanence and the success or failure of the uterine tamponade due to different clinical circumstances.

e. To know the level of success of uterine tamponade.

\section{Methods}

A retrospective, transversal in a series of cases in patients that the SSB was applied for the bleeding control.

\section{Place of the study}

This study was realized in the High Specialty Unit Number 23 of the Mexican Institute of Social Security (IMSS) in Monterrey, Nuevo Leon Maternity Ward, patients that had vaginal birth or cesarean delivery and that postpartum hemorrhage was identified and treated with the SSB UTB. 


\section{Selection criteria}

\section{Inclusion criteria}

i. Patients that gave birth in this unit

ii. Patients that were treated with SSB UTB.

\section{Exclusion criteria}

a. Patients that did not gave birth in this unit.

b. Patients taken to another unit

c. Patients with identified coagulopathy

d. Patients with suspected Triple I (Intra-amniotic, Inflammation and Infection)

\section{Elimination criteria}

i. Files that were not found on the clinical archive

ii. Incomplete Files

\section{Size of the sample}

Patients of female gender older than 18 years of age that gave birth in the unit. The estimation of the sample was realized considering the number of doses to observe, counting with reported incidence of postpartum hemorrhage up to $10 \%$ (P1) of the cases, to observe with a margin of precision of $40 \%$ (E) and a confidence interval of $95 \%$, a sample of 149 patients with SSB UTB was calculated.

\section{Sampling technique}

Not probabilistic, of convenience.

\section{Statistical analysis \\ Tools

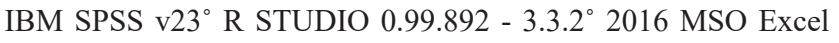 16.0 .6925}

Initial analysis: Central tendency values were determined, standard deviation, analysis of normality and frequency histograms for quantitative variables. The proportion of frequency, total entry percentage and frequency of proportion for categorical variables scales.

Sample analysis: Demographic data and clinical background that prevailed in sample that may be of interest.

Comparative analysis: The patients were grouped in the variety of application and success of the SSB, from this point of comparison the relation between the parametric variables and non-parametric variables were studied. For central tendency variables $T$ Student adjusted to normality of two tails were used to compare the groups of interest to take in account as significant values $P$ less than 0.05 , in case of not being parametric it was studied with a pertinent test according to the number of categories present in the variable. Other results were interpreted by the author reporting interesting data for the study. In case of requiring the MANOVA for multiple variables to compare was made.

For categorical sampling to compare a Fisher of 2 tails was made to describe the differences between the groups of comparison, a significant $\mathrm{P}$ less than 0.05 was accounted.

\section{Results analysis}

Registries of 149 clinical files were studied in which the SSB was applied for postpartum hemorrhage in a comprehended period between 2013-2014.

Among the demographic factors (Table 1), it was observed that the age of the patients with success on the application was less than in those that success was not achieved with 27.23 years of age $(\mathrm{P}=0.035)$, equally with a less quantity of pregnancies with $2.28(\mathrm{P}=0.04)$ in average (Graphics 1-3). A success rate of 9.2.62\% to control the bleeding with the SSB UTB was found $(\mathrm{P}=<0.001)$, additionally a mean time of permanence was of 20.23 hours in success patients (+- 6.27, $\mathrm{P}=0.496)$ and a mean of 18.91 hours in patients in which bleeding control was not obtained with this method (Table 2). The cut point of the time of permanence was analyzed and can predict the success in postpartum hemorrhage patient and it was estimated $($ AUROC $=0.578)$ that beyond 20.5 hours (SEN 52.9\% SPE 77.2\%) no greater benefit is observed in the permanence of the balloon, being able to remove securely in this time.

Table I Demography

\begin{tabular}{|c|c|c|c|c|}
\hline & $\begin{array}{l}\text { Control of bleeding } \\
\text { media, DE }\end{array}$ & $\begin{array}{l}\text { No success } \\
\text { media, DE }\end{array}$ & P.val & T-test \\
\hline Age Years & $27.23 \pm 7015$ & $32 \pm 7.44$ & 0.035 & -2.123 \\
\hline Gestures & $2.28 \pm 1.2$ & $3.09 \pm 1.7$ & 0.04 & -2.073 \\
\hline Births & $0.99 \pm 1.17$ & $1.09 \pm 1.22$ & 0.791 & -0.266 \\
\hline Abortions & $0.29 \pm 0.62$ & $0.55 \pm 0.82$ & 0.2 & -1.286 \\
\hline Cesareans & $0.99 \pm 1.02$ & $1.45 \pm 1.57$ & 0.184 & -1.336 \\
\hline
\end{tabular}

Table 2 Parameter of the SSB Ball

\begin{tabular}{lllllll}
\hline & & \multicolumn{2}{l}{$\begin{array}{l}\text { Control of bleedingNo success } \\
\text { media, DE }\end{array}$} & m.val & t-Test \\
\hline Clogging Time & hrs & $20.23 \pm 6.27$ & $18.91 \pm 4.76$ & 0.496 & 0.683 \\
Lined up & $\mathrm{ml}$ & $407.07 \pm \mathrm{II} 8.94$ & $406.36 \pm 100.63$ & 0.985 & 0.019 \\
Sewer system & $\mathrm{ml}$ & $120.36 \pm 57.53$ & $253.64 \pm 128.94$ & $<0.00 \mathrm{I}$ & -6.552 \\
Gestation weeks & smn & $34.39 \pm 8.86$ & $28.26 \pm 10.35$ & 0.166 & $\mathrm{I} .413$ \\
\hline
\end{tabular}

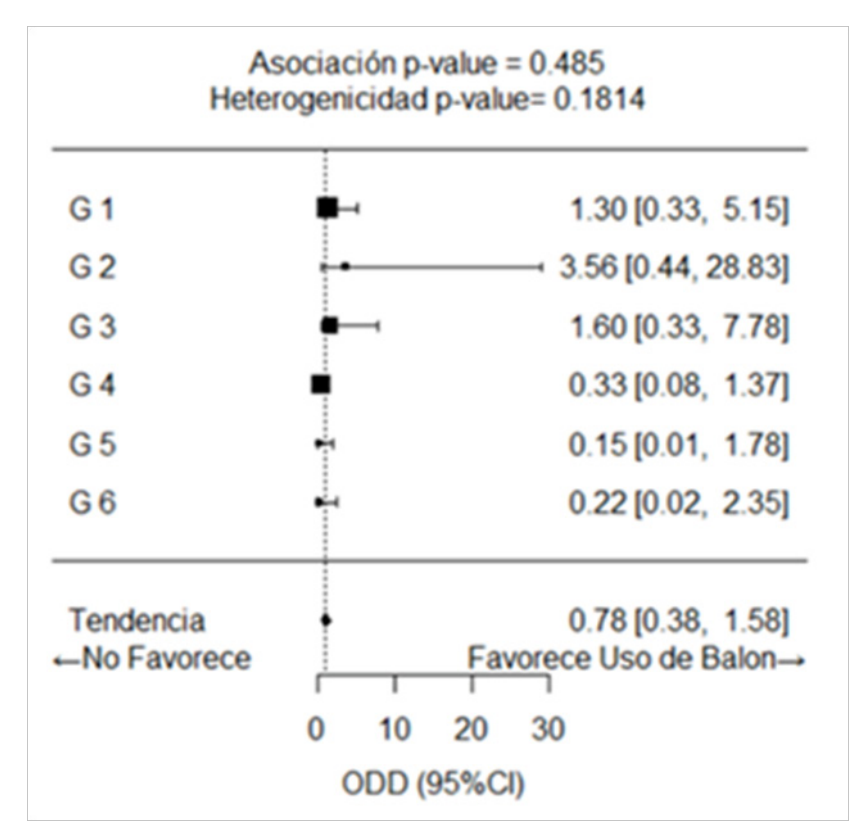

Graphic I Age of the patients is equally with a less quantity of pregnancies with in average. 


\section{Asociación p-value $=0.5257$ Heterogenicidad $p$-value $=0.329$}

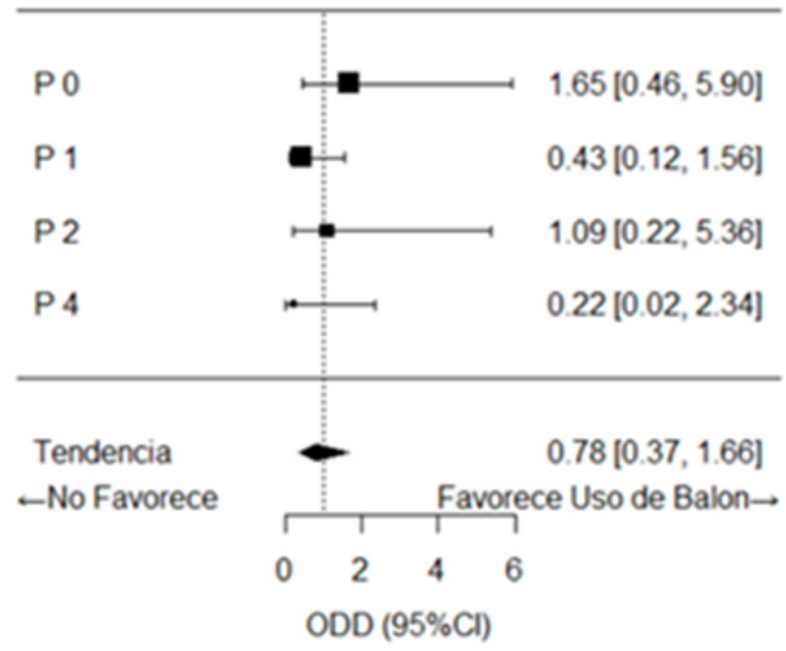

Graphic 2 Age of the patients is equally with a less quantity of pregnancies with in average.

Asociación p-value $=0.3722$

Heterogenicidad $p$-value $=0.0842$

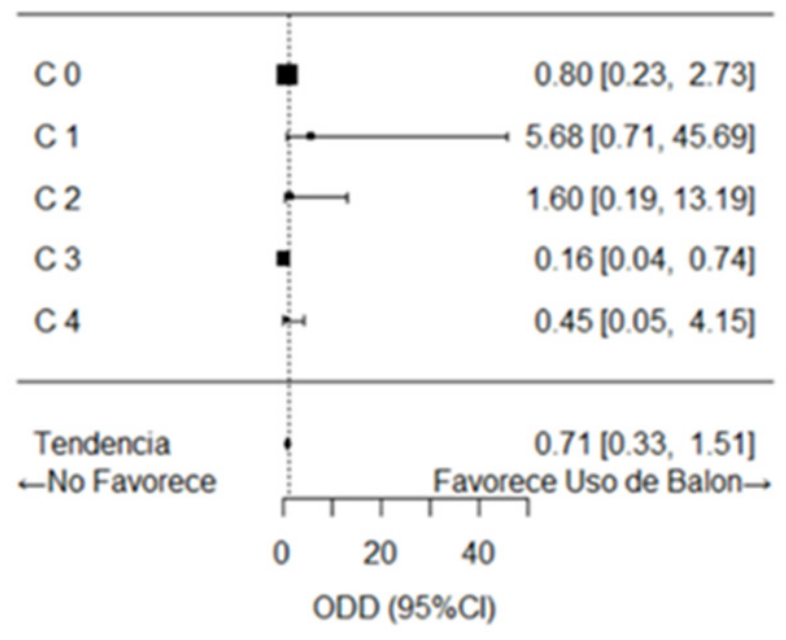

Graphic 3 Age of the patients is equally with a less quantity of pregnancies with in average.

We found in our study that the maternal age being less than 30 years of age was an increasing factor in the incidence of success in the uterine tamponade with SSB. In our sample we found that the incidence of success in the patients was relatively high, in the literature the incidence of success with the Bakri SOS Balloon was reported between $87.5-90 \%$, until this moment there is no study that has explored extensively the time of permanence and uterine balloon tamponade, in our study we wanted to find the mapping of events, the time in which no greater benefit would be registered in matter of permanence, several author have reported a range of time of permanence between 10 minutes and 48 hours, without making specific reference in time of the success cases.

\section{Discussion}

The number of cases in this report is bigger than the majority of other studies of uterine tamponade with different kinds of balloons. We could observe clearly that the age of the patients is a determinant factor for the success of the tamponade, the average in our study was of 27.23 years of age, in the cases in which the treatment failed, the mean age was higher. Lohano et al. ${ }^{1}$ reported a mean age of 26 years in the 10 cases reported by Sagai et al. ${ }^{2}$ a mean of 30 years was found, so the maternal age less than 30 years is a crucial factor that can indicate the probable success of uterine tamponade with SSB.

Other characteristic that were found in the patients that presented success in the application of the SSS UTB was the number of pregnancies with a 2.28 average, in comparison with the patients that success was not achieved, which were mainly multigravida, similarly to the study published by Aibar et al. ${ }^{3}$ where $58 \%$ of the patients with this treatment were nulliparous, also in the Nagai group were patients with 1 or 2 pregnancies, in the Gronval ${ }^{10}$ the majority of the patients were in their first pregnancy, for which can be concluded that the less number of pregnancies is a factor that increases the success rate in SSB UTB.

The success rate in our study was $92.62 \%$, higher than the literature reports, where a $87.5-90 \%$ success range is handled, Nagai et al. ${ }^{2}$ ] found a success rate of $100 \%$ when atony was the cause of $\mathrm{PPH}$, in Bakri's original study with 5 patients with placenta Previa a success rate of $100 \%$ was obtained, it should be noted that our study is one of the first with Sengstaken Blakemore Balloon published, the majority of the known studies until this moment use Bakri Balloon for uterine tamponade.

The medium time of permanence in success cases was of 20.23 hours and in the cases in which success was not achieved was of 18.91 hours, in the published series until this moment it is not analyzed this category in relation of the success or failure of the uterine tamponade, Gronval $^{10}$ reported an average of time of 12-24 hours, Nagai et al. ${ }^{2}$ with his Bakri Balloon study published a range of permanence of 24 hours; Aibar (2013) also with the Bakri Balloon reported a range of 14-21 hours, in the study from Lohano et al. ${ }^{1}$ in which they used a condom (Bangladesh) associated with a catheter as uterine balloon tamponade, the medium time of permanence was of 22 hours, in the cohort of Reynosa et al. ${ }^{7}$ that was carried out with Sengstaken Blakemore the average time was of 20 hours. Analyzing this data. It was estimated that beyond 20.5 hours no greater benefit was offered in the permanence of the uterine tamponade, so that it can be removed securely in this time.

\section{Conclusion}

The SSB Balloon as uterine tamponade is being used in Nuevo Leon, because it has a better cost-benefit for us. Nevertheless, is mandatory to carry out more studies to confirm this relation and demonstrate that it can be removed in less time. Because our study has a better sample number than the majority of previous studies published, based on this study it can be concluded that it can be safely removed in a period no more than 20.5 hours.

\section{Acknowledgements}

None.

\section{Conflicts of Interest}

\section{None.}




\section{References}

1. Lohano R, Haq G, Kazi S, et al. Intrauterine balloon tamponade for the control of postpartum haemorrhage. J Pak Med Assoc. 2016;66(1):2226.

2. Nagai S, Kobayashi H, Nagata T, et al. Clinical Usefulness of Bakri Balloon Tamponade in the Treatment of Massive Postpartum Uterine Hemorrhage. Kurume Med J. 2015;62(1-2):17-21.

3. Aibar L, Aguilar MT, Puertas A, et al. Bakri balloon for the management of postpartum hemorrhage. Acta Obstet Gynecol Scand. 2013;92(4):465467.

4. Pendleton AA, Natarajan A, Ahn R, et al. A qualitative assessment of the impact of a uterine balloon tamponade package on decisions regarding the role of emergency hysterectomy in women with uncontrolled postpartum haemorrhage in Kenya and Senegal. BMJ Open. 2016;6:e010083.

5. Érika Fonseca Chimá. Bakri ball in postpartum hemorrhage in Medellín (Colombia): case report and literature review. Rev Colomb Obstet Ginecol. 2010;61(4):335-340.

6. Antonio Morales L, Alvaro Insunza F, Rodrigo Latorre R. Successful management of postpartum haemorrhage by plaortous aorism by tamponade with bakri balloon. Rev Chil Obstet Ginecol. 2006;71(2):121124

7. Yolanda Reynosa Oviedo. Efficacy and safety of uterine tamponade for hemorrhage control and obstetric hysterectomy reduction. historical cohort in nuevo león, mexico, 2013. Revista Colombiana de Obstetricia y Ginecología. 2015;66(3):186-194.
8. Georgiou C. Intraluminal pressure readings during the establishment of a positive 'tamponade test' in the management of postpartum haemorrhage. BJOG. 2010;117(3):295-303.

9. Ajayi OA, Sant M, Ikhena S, et al. Uterine rupture complicating sequential curettage and Bakri balloon tamponade to control secondary PPH. BMJ Case Rep. 2013.

10. Gronvall M, Tikkanen M, Tallberg E, et al. Use of Bakri balloon tamponade in the treatment of postpartum hemorrhage: a series of 50 cases from a tertiary teaching hospital. Acta Obstet Gynecol Scand. 2013;92(4):433-438.

11. Tindell K, Garfinkel R, Abu-Haydar E, et al. Uterine balloon tamponade for the treatment of postpartum haemorrhage in resource-poor settings: a systematic review. BJOG. 2013;120(1):5-14.

12. Say L, Chou D, Gemmill A, et al. Global causes of maternal death: a WHO systematic analysis. Lancet Glob Health. 2014;2(6):e323-e333.

13. Joshi V, Otiv S, Majumder R, et al. Internal iliac artery ligation for arresting postpartum haemorrhage. BJOG. 2007;114(3):356-361.

14. Kayem G, Kurinczuk J, Alfirevic Z, et al. Specific second-line therapies for postpartum haemorrhage: a national cohort study. BJOG. 2011;118(7):856-864.

15. Kavak SB, Kavak EÇ, Demirel I, et al. Double-balloon tamponade in the management of postpartum hemorrhage: a case series. Ther Clin Risk Manag. 2014;10:615-620. 